\title{
VEGF antagonism reduces edema formation and tissue damage after ischemia/reperfusion injury in the mouse brain
}

\author{
Nicholas van Bruggen, ${ }^{1}$ Harold Thibodeaux, ${ }^{2}$ James T. Palmer, ${ }^{1}$ Wyne P. Lee, ${ }^{3}$ \\ Ling Fu, ${ }^{3}$ Belinda Cairns, ${ }^{4}$ Daniel Tumas, ${ }^{4}$ Robert Gerlai, ${ }^{1}$ Simon-Peter Williams, ${ }^{1}$ \\ Menno van Lookeren Campagne, ${ }^{3}$ and Napoleone Ferrara ${ }^{2}$
}

${ }^{1}$ Department of Neuroscience,

${ }^{2}$ Department of Cardiovascular Research,

${ }^{3}$ Department of Immunology, and

${ }^{4}$ Department of Pathology, Genentech Inc., South San Francisco, California 94080, USA

Address correspondence to: Nicholas van Bruggen, Genentech Inc., Department of Neuroscience (MS\#72), 1 DNA Way, South San Francisco, California 94080, USA.

Phone: (650) 225-6057; Fax: (650) 225-5688; E-mail: vanbruggen.nick@gene.com.

Received for publication August 20, 1999, and accepted in revised form October 18, 1999.

VEGF is mitogenic, angiogenic, and a potent mediator of vascular permeability. VEGF causes extravasation of plasma protein in skin bioassays and increases hydraulic conductivity in isolated perfused microvessels. Reduced tissue oxygen tension triggers VEGF expression, and increased protein and mRNA levels for VEGF and its receptors (Flt-1, Flk-1/KDR) occur in the ischemic rat brain. Brain edema, provoked in part by enhanced cerebrovascular permeability, is a major complication in central nervous system pathologies, including head trauma and stroke. The role of VEGF in this pathology has remained elusive because of the lack of a suitable experimental antagonist. We used a novel fusion protein, $\mathrm{mFlt}(1-3)-\mathrm{IgG}$, which sequesters murine VEGF, to treat mice exposed to transient cortical ischemia followed by reperfusion. Using high-resolution magnetic resonance imaging, we found a significant reduction in volume of the edematous tissue 1 day after onset of ischemia in mice that received $\mathrm{mFlt}(1-3)-\mathrm{IgG}$. 8-12 weeks after treatment, measurements of the resultant infarct size revealed a significant sparing of cortical tissue. Regional cerebral blood flow was unaffected by the administration of mFlt(1-3)-IgG. These results demonstrate that antagonism of VEGF reduces ischemia/reperfusion-related brain edema and injury, implicating VEGF in the pathogenesis of stroke and related disorders.

J. Clin. Invest. 104:1613-1620 (1999).

\section{Introduction}

Brain edema is a major and often life-threatening complication of a variety of brain injuries. Clinically, late stage edema, as defined by the disruption of the bloodbrain barrier, is the most common form of brain edema (1). A number of physiological conditions need to be present for the formation of cerebral edema, including altered cerebrovascular permeability, hydrostatic pressure, and appropriate osmotic and colloidal gradients. Increased cerebrovascular permeability is, however, the single most important factor for the development of brain edema.

VEGF is a potent mediator of vascular permeability (2-4). VEGF is responsible for the plasma extravasation associated with tumor microvasculature, and its effect on dermal microvessels is much greater than any other known mediators of vessel permeability (e.g., histamine) (5). Diffuse VEGF mRNA expression has been observed in adult rat brain, but at relatively low abundance (6). However, reduced oxygen tension is a known trigger for VEGF expression (7), and enhanced levels of VEGF and its receptors, Flt- 1 and Flk-1/KDR, were shown to be induced in the rat brain after focal cerebral ischemia (8-10). Similar expression has recently been shown to occur in the human brain after ischemic stroke (11). The role of VEGF in the pathogenesis of stroke, and the formation of cerebral edema is unclear with contradictory experimental observations cited in the literature. For example, Nag et al. (12) used a cortical cold-injury rat model to demonstrate the presence of murine VEGF in permeable pial vessels and arterioles within the damaged tissue, and, from this observation, they inferred that VEGF is 1 of several factors that may mediate edema formation. On the other hand, Hayashi et al. (13) reported that VEGF itself, when applied topically to the surface of a reperfused rat brain after transient cerebral artery occlusion, reduced ischemic brain damage, infarct volume, and edema formation.

To date, the role of VEGF in the pathophysiology of cerebral infarction remains unknown. Because the majority of research studies are performed on rodents, the lack of a suitable pharmacological antagonist effective in either rat or mouse prevents a clear understanding of the contribution of VEGF in the patho- 
genesis of stroke and related disorders. Recently, however, Ferrara et al. (4) took advantage of the high affinity and cross-reactivity of VEGF binding to the tyrosine kinase receptor, Flt-1 $(14,15)$. They demonstrated that the truncated receptor, consisting of the first 3 immunoglobulin-like domains of the murine Flt-1 fused to a Fc-IgG from the mouse, $\mathrm{mFlt}(1-3)-\operatorname{IgG}$, is an effective inhibitor of rat and mouse VEGF. Furthermore, this fusion protein has an adequate systemic half-life to be suitable for in vivo efficacy studies. Using this fusion protein to sequester VEGF, we have been able to show that VEGF is partly responsible for the cortical edema formation in a model of focal ischemic injury with reperfusion.

\section{Methods}

Protocol. Mice (C57BL-6J, male; The Jackson Laboratory, Bar Harbor, Maine, USA) weighing 18-25 g were used in this study. A total of 54 mice were included in this study. Of these, 36 were used to establish acute and long-term efficacy by magnetic resonance imaging (MRI) and were examined at both 24 hours and again 8-12 weeks after ischemia.

A separate study was designed to investigate the effects of $\mathrm{mFlt}(1-3)-\mathrm{IgG}$ on local cerebral blood flow (CBF) changes during ischemia. An additional 18 animals were included in this study, and the changes in $\mathrm{CBF}$ were measured during the occlusion with laser Doppler (as described below). These animals were examined by MRI at the 24-hour time point only.

The protocol, housing, and anesthesia concurred with guidelines and were approved by Genentech's IACUC (Institutional Animal Care and Use Committee), in compliance with AAALAC (Association for Assessment and Accreditation of Laboratory Animal Care) regulations. In the efficacy study, the animals were randomly divided into 2 groups and given either mFlt (1-3)-IgG (10 mg/kg, intraperitoneally in PBS) or an irrelevant control IgG (anti-gp-120) (10 mg/kg, intraperitoneally in PBS) at approximately $12-16$ hours before surgery, after reperfusion, and again 1 day after surgery and before the MRI examination. In the second set of experiments, designed to monitor changes in relative blood flow during occlusion,

Figure 1 \pm SEM; $\left.{ }^{*} P<0.05\right)$.
mFlt(1-3)-IgG was given 30 minutes before occlusion to achieve near-maximal plasma levels of the fusion protein, and to therefore increase our chance of observing any blood flow response. In both studies, T2-weighted MRI performed 24 hours after the onset of ischemia assessed the degree of edema formation. The eventual size of the infarction was assessed 8-12 weeks later using high-resolution anatomical MRI. After the final MRI exam, the animals were perfused and fixed for histology as described below.

Surgical procedure. Gaseous anesthetic (1.0-2.0\% isoflurane in a mixture of $70 \%$ nitrous oxide and $30 \%$ oxygen) was used for all surgical procedures. Physiological parameters, including heart rate, respiration, and core temperature were monitored throughout the experiment and remained within an acceptable range. Occlusion of the middle cerebral artery (MCA) was performed using a modification of the technique described in detail elsewhere (16). In brief, after the temporalis muscle was incised and retracted to expose the squamous bone, a craniotomy $(1.5 \mathrm{~mm}$ in diameter) was performed at the juncture of the zygoma and squamous bones to localize the right MCA. The MCA was ligated with an 11-0 Dermalon suture (Davis \& Geck, Division of American Cyanamid Company, Wayne, New Jersey, USA). The ipsilateral common carotid artery (CCA) was

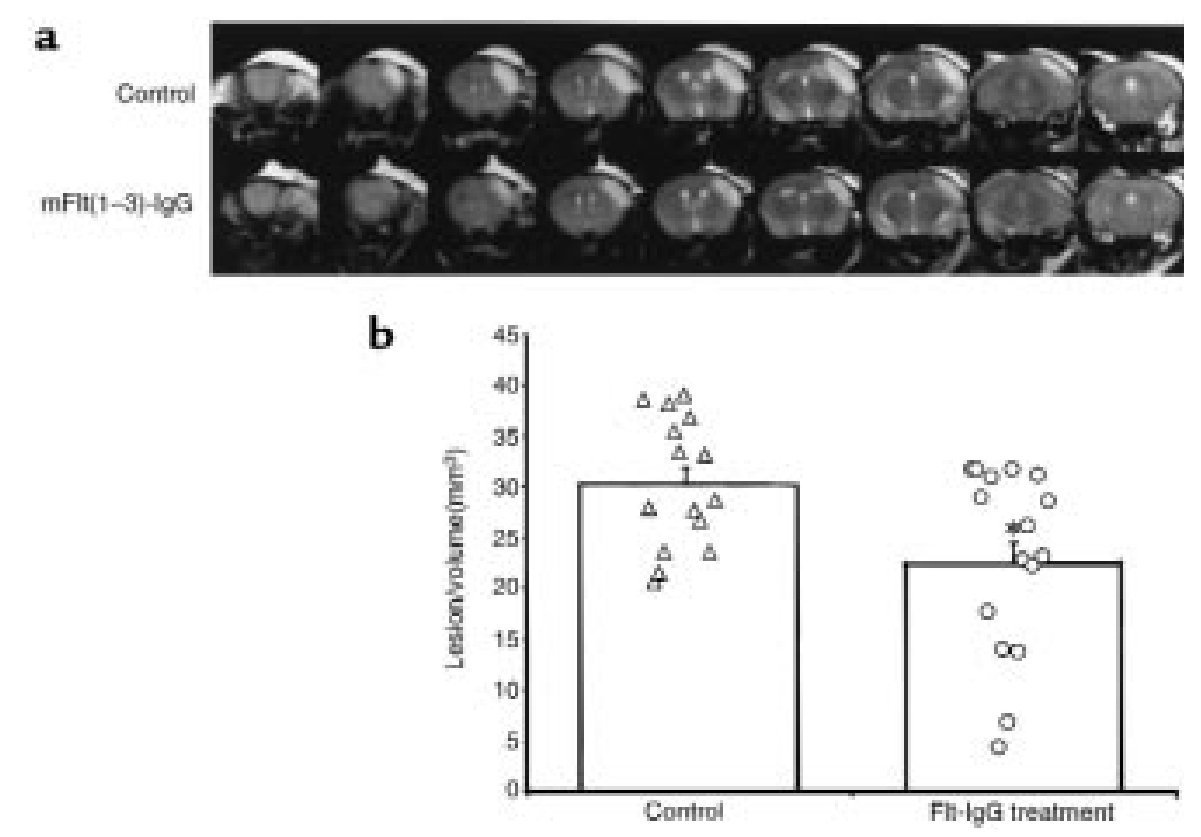

mFlt(1-3)-IgG treatment reduction of cortical edema volume is demonstrated by T2-weighted MRI. (a) Representative MRI data for the 2 mice closest to the mean for each group are shown. A series of 1-mm contiguous coronal slices were made covering the entire mouse brain. The cortical edema, as defined by the region of 2 hyperintensity, is reduced with $\mathrm{mFIt}(1-3)$ - IgG treatment (bottom row) compared with control group (top row). The area of high signal intensity above the skull is due to inflammation associated with the surgical procedure. The volume of edematous tissue is clearly delineated by the region of high signal intensity within the cerebral cortex, and, in the control animal, extends from the frontal cortex caudally to almost the full extent of the cerebral cortex. (b) $\mathrm{mFlt}(1-3)-\lg G$ produced a significant reduction in the lesion volume, as measured from the T2weighted MRI (data from the individual animals are shown together with the mean lesion volume 

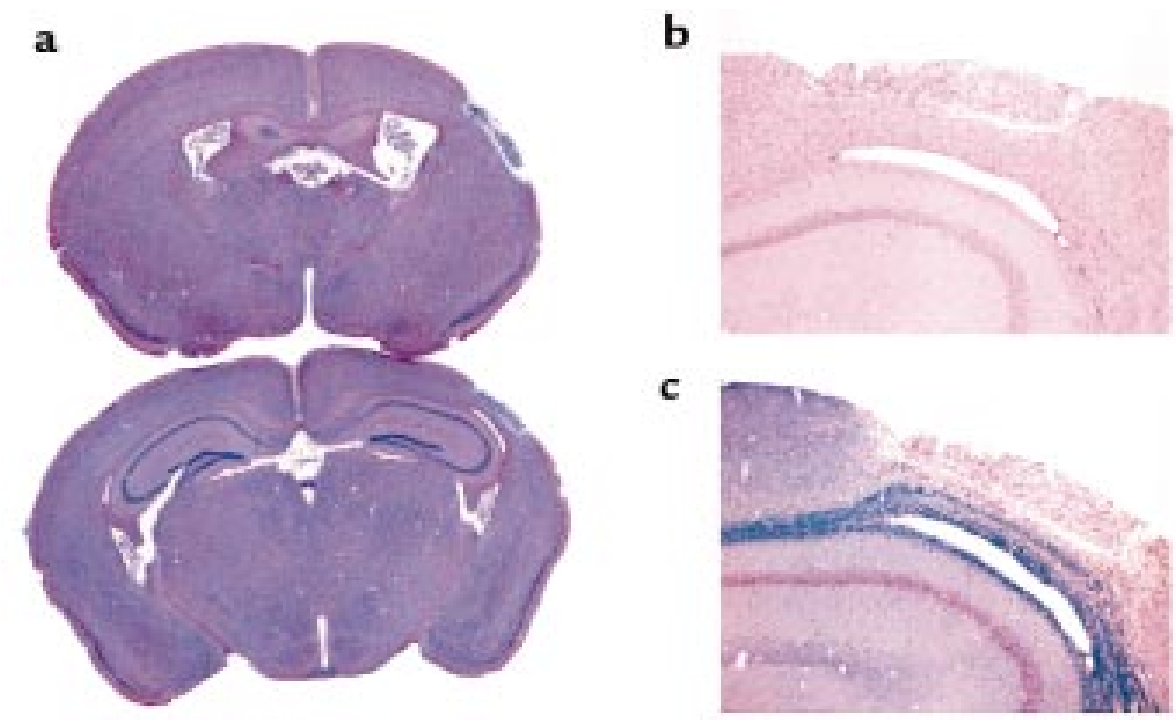

\section{Figure 2}

Histology revealed a discreet cortical lesion with cavitation and gliosis several months after ischemia/reperfusion injury. Temporary ligation of the MCA in this model induced a consistent focal lesion. In each animal, evaluated 8-12 weeks after injury, focal infarction induced discrete cavitation and loss of a unilateral focal area of tissue in the cerebral cortex involving the somatosensory cortex, predominantly the S1 forelimb and barrel fields with extension into the secondary somatosensory cortex. (a) The extent of tissue damage is seen from the lowmagnification H\&E sections located approximately $0.5 \mathrm{~mm}$ anterior and $2 \mathrm{~mm}$ posterior to Bregma. The motor cortex was unaffected. This cortical lesion was typically full thickness and extended from the external capsule with corpus callosum as the inner limit and the meninges as the outer limit. (b) Higher magnification H\&E section. (c) The Cresyl violet and Luxol fast blue section. In each animal, the infarcted area was a cavitary lesion that contained a dense population of foamy macrophages (gitter cells), rare neutrophils, and few stromal elements that supported small vessels. The small vessels within the lesion commonly had a mild perivascular infiltrate of lymphocytes. With the exception of a mild increase in glial cells and occasional necrotic neurons in the immediately adjacent neuropile, the discrete infarct was bordered by histologically normal tissue, including the adjacent cerebral cortex and the underlying caudate putamen.

also exposed. Focal ischemia was achieved by tandem occlusion of the MCA and by placing a micro-aneurysm clip (Roboz, Surgical Instrument Company Inc., Rockville, Maryland, USA) on the CCA. The vessels remained occluded for either 30 or 45 minutes, at which time the blood flow to the MCA territory was restored, first by removing the aneurysm clip and then the suture. Successful restoration of blood flow was assessed under the operating microscope. Five mice were excluded from the study because of unsuccessful occlusion or reperfusion. During the recovery period, the animals were continuously warmed on a thermoregulated blanket (Harvard Apparatus Co., South Natick, Massachusetts, USA) before being returned to their normal housing.

MRI. Twenty four hours after the onset of ischemia, the animals were re-anesthetized $(1.0 \%$ isoflurane in oxygen) and placed in a purpose-built plastic head holder (Applied Neuroscience, Thurso, United Kingdom). The head was held rigid so that the parietal surface of the skull was horizontal in the magnet, and the brain was centered in a volume radiofrequency coil (Morris Instruments Inc., Gloucester, Canada) operating in quadrature mode (internal diameter, $4 \mathrm{~cm}$ ). The animals' core temperature was monitored and maintained at $37 \pm 1^{\circ} \mathrm{C}$ with warm air. The MRI experiments were performed on a 4.7-T Varian Inova MRI system (Varian Inc., Palo Alto, California, USA). Correct positioning of the animal and slice planning were confirmed from a series of scout images obtained in the coronal plane.
To assess the degree of cerebral edema, high-resolution MRI was performed using a fast-spin echo sequence with T2 weighting. Imaging parameters include: effective echo time (effTE) of 60 milliseconds; repetition rate of 4 seconds; field of view (FOV) was 20 $\mathrm{mm} ; 128 \times 128$ matrix; number of excitation (NEX) was 8; echo train length was 16; and slice thickness was 1 $\mathrm{mm}$, giving in-plane resolution of approximately 150 $\mu \mathrm{m}$. Accurate assessment of the eventual infarct size was obtained from high-resolution anatomical MRI using the same protocol but at higher resolution and signal to noise (effTE of 8 milliseconds; NEX was 64; slice thickness was $0.5 \mathrm{~mm}$; FOV was $16 \mathrm{~mm}$ ). For these studies a smaller volume radiofrequency coil with an internal diameter of $2 \mathrm{~cm}$ was used.

Preparation of $m$ Flt (1-3)-IgG. The $\mathrm{mFlt}(1-3)-\operatorname{IgG}$ was constructed by PCR amplification of a mouse 17-day embryo cDNA (CLONTECH Laboratories Inc., Palo Alto, California, USA) as described by Ferrara et al. (4). Recombinant mFlt(1-3)-IgG was purified from media conditioned by transfected Chinese hamster ovary cells and characterized as reported previously (4).

Laser Doppler measurements of relative CBF. Cortical CBF was measured before and after MCA occlusion by a laser Doppler microvascular perfusion monitor (PeriFlux system 4000; Perimed Inc., North Royalton, Ohio, USA). After the craniotomy and the localizing of the MCA, a 1-mm stainless steel probe was placed on the right parietal bone perpendicular to the part of 


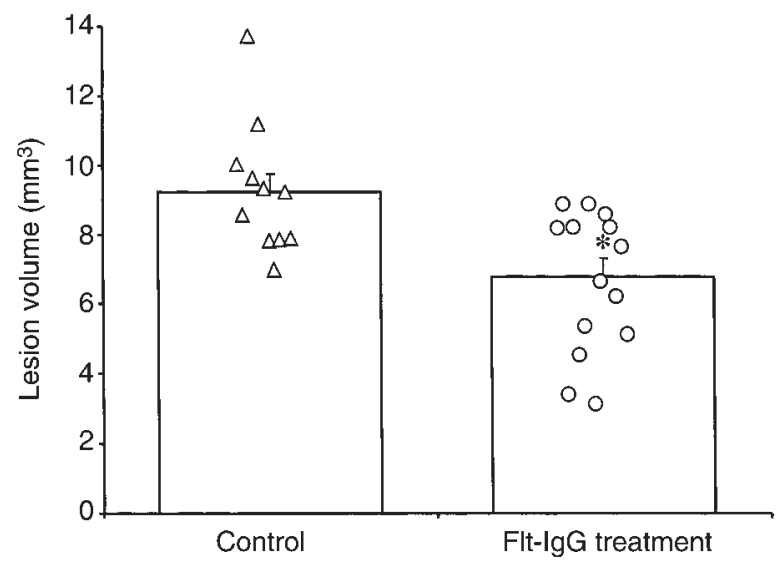

Figure 3

mFlt-(1-3)-IgG treatment affords long-term tissue salvage as demonstrated by high-resolution anatomical MRI. The size of the infarct can be readily delineated from the coronal MRI sections by measuring the area of the remaining cortex and comparing it with the contralateral hemisphere. Using this approach, the size of the cortical damage was found to be significantly reduced in the $\mathrm{mFIt}(1-3)$-IgG-treated group compared with control group $\left({ }^{*} P<0.01\right)$.

cortex supplied by MCA. Relative blood flow was monitored at 3 points as illustrated in Figure 5. Position $\mathrm{A}$ was $4 \mathrm{~mm}$ posterior to coronal suture, and 4.5 $\mathrm{mm}$ lateral to sagittal suture; position B was $2.5 \mathrm{~mm}$ posterior to coronal suture, and $4 \mathrm{~mm}$ lateral to saggital suture; and position $\mathrm{C}$ was $1 \mathrm{~mm}$ posterior to coronal suture and $3.5 \mathrm{~mm}$ lateral to sagittal suture. After the baseline measurement of $\mathrm{CBF}$ has been established, the MCA and ipsilateral CCA were occluded and a postischemic CBF was recorded 10-15 minutes after onset of ischemia. The vessels remained occluded for 30 minutes, at the end of which time the animals were allowed to recover for the MRI exam 24 hours later. A single dose of $\mathrm{mFlt}(1-3)-\mathrm{IgG}(10 \mathrm{mg} / \mathrm{kg}$, intravenously in PBS) was given 30 minutes before occlusion of the MCA.

Histology. At 12 weeks after infarct, the mice were anesthetized using sodium pentobarbital and perfused via intracardiac cannulation with $20 \%$ neutral-buffered formalin. The brains were removed and placed in $20 \%$ neutral-buffered formalin for an additional 4 days. Subsequently, using a mouse brain matrix mold, the brains were serially sectioned into 2 -mm slices and processed for paraffin embedding. Paraffin sections (3 $\mathrm{mm}$ ) were obtained from each slice and were stained with hematoxylin and eosin (H\&E) and Luxol Fast blue with Cresyl violet by standard methods. The latter stain was used in the estimation of cortical infarction because it allowed clear demarcation of the cerebral cortex gray matter from the underlying white matter tract boundaries, including the external capsule and the corpus callosum.

Image analysis. Image data analysis and post-processing were performed off-line using image analysis software (MRVision Co., Menlo Park, California, USA).
The extent of the edema formation was obtained by delineating the regional cortical hyperintensity by eye from the T2-weighted MRI data obtained 24 hours after ischemia (a single experimenter who was blinded to the groupings performed the data analysis). The degree of infarction was estimated from the high-resolution anatomical images obtained at 8-12 weeks of reperfusion by establishing the volume of unaffected cortex and comparing it with the contralateral hemisphere. To estimate the infarct size from histology, 4 sections from each animal were captured using a Spot cooled color digital camera (Diagnostic Instruments Inc., Sterling Heights, Michigan, USA) mounted on a Nikon dissection microscope with a $\times 1$ lens. The sections were analyzed using MRVision Co. software. The cortical tissue was identified with the assistance of a mouse brain atlas (17). Different measurements were made and integrated over the four slices.

\section{Results}

To investigate the physiological role of VEGF in the pathogenesis of stroke we chose a murine model of focal cerebral ischemia with reperfusion. Occlusion of the right MCA together with the right CCA with reperfusion produces a reproducible and discrete focal infarct in the cerebral cortex (16). In this model, delayed tissue damage secondary to the transient arterial occlusion is found with profound tissue reactivity and edema occurring 1-2 days after the ischemia insult, and its severity depends upon the duration of occlusion (18). The extent of the edematous tissue can be visualized using T2-weighted, fast-spin echo MRI sequence obtained 24 hours after the onset of ischemia. As illustrated in Figure 1, a pronounced hyperintensity on the $\mathrm{T} 2$-weighted scan was evident at 24 hours. It covered a large volume of the right cortex, extending from the primary motor cortex in the forebrain and as far as the interaural line and included the primary visual cortex. The edematous changes were confined to the cortex and did not extend beyond the corpus callosum. The lesion volume, as defined by the region of high signal intensity within the ischemic cortex, was greater after 45 minutes of ischemia than it was after 30 minutes $\left(30.5 \pm 1.6 \mathrm{~mm}^{3}\right.$ and $22.5 \pm 3.4$ $\mathrm{mm}^{3}$, mean lesion volume \pm SEM, for 45 - and 30 minute occlusion times, respectively; $P<0.05$ ). This is consistent with the notion that early reperfusion is beneficial despite the occurrence of secondary injury associated with reflow. Representative T2-weighted MRI (i.e., the animal closest to the mean for each group) recorded 24 hours after 45 minutes of focal ischemia are shown in Figure 1. There is a clear reduction in the extent of the cerebral edema associated with $\mathrm{mFlt}(1-3)-\mathrm{IgG}$ treatment compared with the control group. The volume of the edematous tissue was quantified, and the results are shown in Figure $1 \mathrm{~b}$. Administration of mFlt(1-3)-IgG caused a significant reduction in the volume of cerebral edema as defined by the region of hyperintensity on the T2-weighted 
a
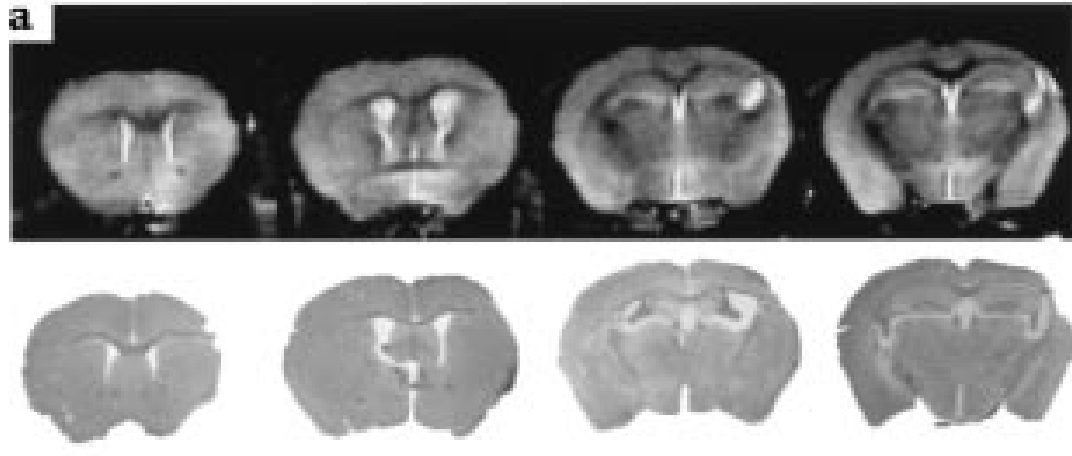

b
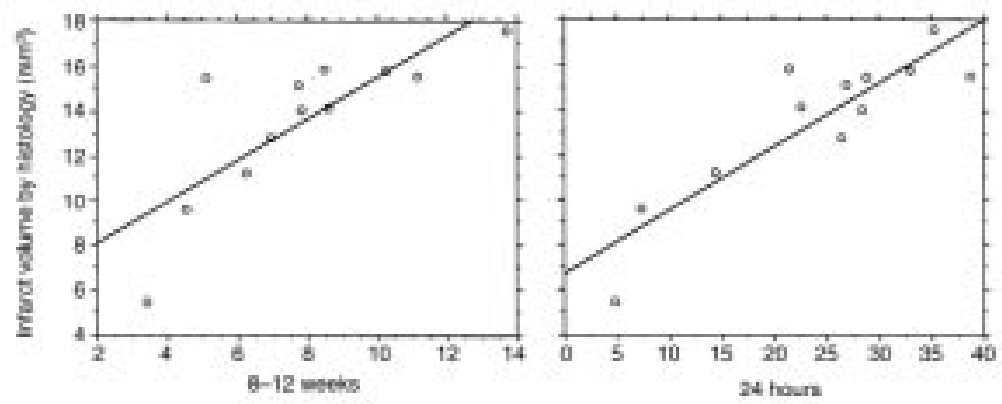

Figure 4

The histological measure of the infarct size correlates to MRI predictors of infarction. (a) The appearance of the cortical infarction by histology (bottom row), together with the equivalent high resolution MRI (top row) at 8-12 weeks after onset of ischemia. (b) The infarction volume determined by histology was compared with the infarct volume measured with high-resolution, anatomical MRI and the extent of edema as seen from the early T2-weighted MRI. Good correlation between the histology and MRI measurement made at both 8-12 weeks and 24 hours after ischemia/reperfusion was seen.
MRI scan acquired 1 day after onset of ischemia $(\sim 30 \%$ reduction; $P<0.05$, Student's $t$-test; $n=15$ and 16 in control and treatment groups, respectively).

As evident from the histology, this lesion continues to evolve for many days, or possibly weeks, after its onset and eventually produces a core of pan-necrosis and cavitation, with little evidence of selective neuronal death in the surrounding tissue (Figure 2). To establish whether mFlt(1-3)-IgG-mediated reduction of acute cerebral edema conferred long-term salvage of cortical tissue in this model, the surviving animals ( 25 of 31 ; 4 from the control group and 2 from the treated group died) were re-examined between 8 and 12 weeks later. At this time, the final size of the infarct can be deduced from the degree of remaining cortical tissue. This can be determined using high-resolution anatomical MRI that accurately depicts the lesion volume. Again, a fast-spin echo technique was used, but with more signal averaging and reduced T2-weighting to allow for sufficient signal-tonoise for higher resolution studies. Using this imaging sequence, we were able to obtain in-plane resolution of about $125 \mu \mathrm{m}$ with 0.5 -mm-thick slices. The imaging time for this study was about 30 minutes per mouse.

The size of the infarct was determined by delineating the amount of remaining cortex and comparing it with the contralateral hemisphere. The size of the cortical infarction is significantly reduced by the administration of $\mathrm{mFlt}(1-3)-\operatorname{IgG}$ measured 8-12 weeks later (30\% reduction in infarct size $(P<0.01$, Student's $t$-test; $n=11$ and 14 in control and treatment groups, respectively) (Figure 3). There was good agreement between the infarct volume measured by MRI and that determined using conventional histology $(r=0.80, n=12)$ (Figure 4). Fur- ther, from the MRI studies, the severity of tissue edema as measured at 24 hours correlated with size of the infarct measured 8-12 weeks later $(r=0.62, n=12)$ (Figure 4). Accordingly, the treated animals exhibited a reduction in the development of cerebral edema, which may provide further preservation of cortical tissue.

It should be noted that although a reduction in the area of cortical edema, as defined on the T2-weighted images, correlates with long-term preservation of cortical tissue, the extent of tissue affected is much greater than the ultimate size of the infarction. This observation supports the notion that although spontaneous resolution of tissue edema can occur, edema itself may contribute to the pathogenesis of infarction in this model. The discrepancy between the high-resolution anatomical MRI measurement of cortical damage and that measured by histology presumably reflects the difference in the accuracy of each approach and is greater in cases with smaller lesion volumes. MRI lacks the resolution offered by the histological analysis, and errors from inaccuracies in slice thickness and partial volume can confound the measurement.

In a separate study designed to determine the effects of mFlt(1-3)-IgG on cerebral blood flow, laser Doppler was used to record the changes in relative flow during occlusion of the MCA. A reduction in relative blood flow to $5-10 \%$ of the baseline value was seen in all animals examined. There was no significant difference in the mean relative tissue perfusion between the control and mFlt(1-3)-IgG and treated animals in any of the 3 areas examined (Figure 5). To determine the extent of the edematous pathology 


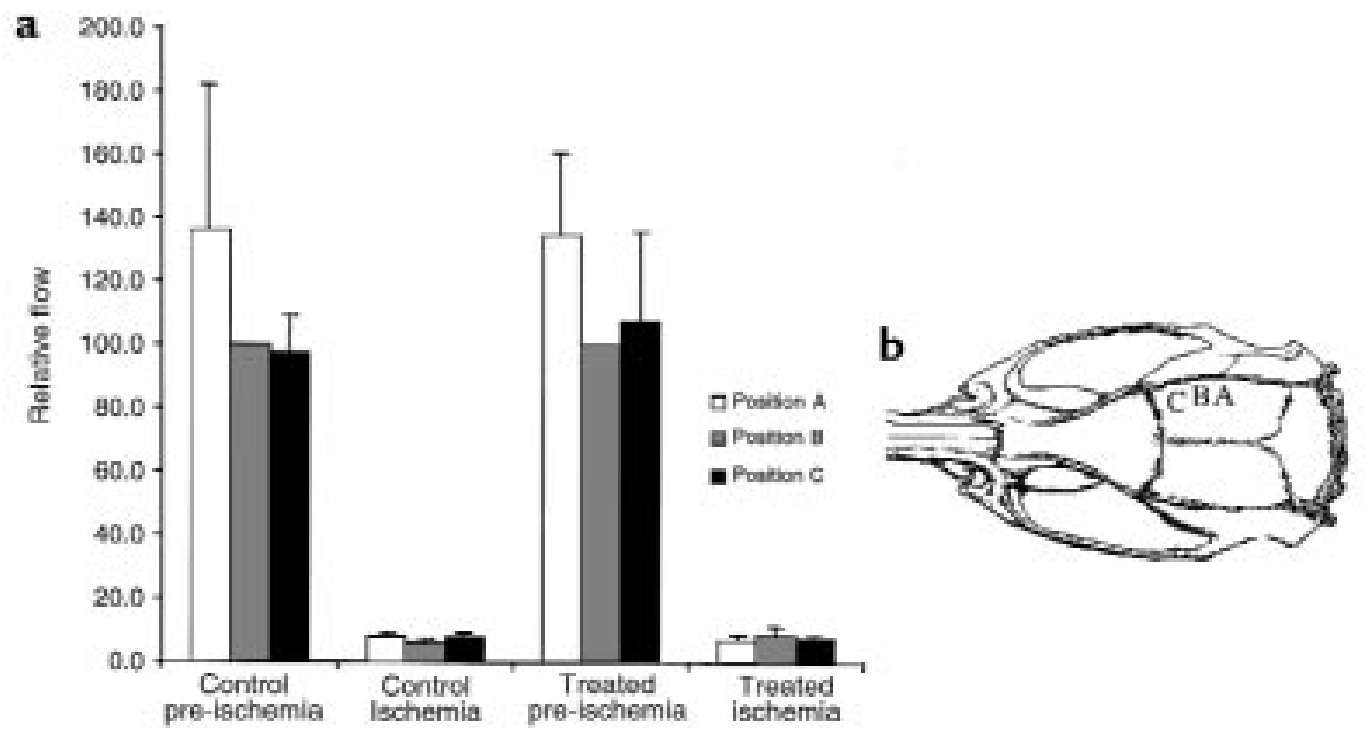

\section{Figure 5}

The relative change in CBF after MCA occlusion was unaffected by pretreatment with mFlt(1-3)-lgG. Relative CBF was measured using laser Doppler flowmetry with a $1.0-\mathrm{mm}$ straight steel probe positioned close to the right parietal bone above the MCA territory. Relative blood flow was monitored before and 10-15 minutes after occlusion of the MCA at the 3 positions shown in $\mathbf{b}$. Relative flow values represent the mean percent change in flow normalized to the pre-ischemic level recorded from position $B$ (mean $\pm S E M)$. A reduction in $C B F$ was seen upon occlusion for all mice examined, but was unaffected by pretreatment with mFlt(1-3)-lgG.

MRI was performed on these animals 24 hours after surgery. A significant reduction in the lesion volume, as defined by the region of $\mathrm{T} 2$ hyperintensity, was seen in this study $\left(22.5 \pm 3.4 \mathrm{~mm}^{3}\right.$ and $5.5 \pm 3.3 \mathrm{~mm}^{3}$ mean lesion volume for control and $\mathrm{mFlt}(1-3)$-IgG-treated groups, respectively; $P<0.01$ ) (Figure 6). These data show that the reduction in the cortical edema, as assessed by T2-weighted MRI, afforded by pretreatment with the fusion protein is not mediated by a change in the $\mathrm{CBF}$ during ischemia. Further, a single dose of mFlt(1-3)-IgG in this model of mild transient ischemia is efficacious.

\section{Discussion}

The formation of cerebral edema and central nervous system (CNS) inflammation are often a late stages in the pathogenesis of infarction resulting from cerebral ischemia. The beneficial effects of prompt and adequate restoration of blood flow after an acute ischemic event may be undermined by reperfusion injury to the cerebral microvasculature comprising the cerebral microcirculation, and thus contribute to the formation of cerebral edema and the potentially fatal developments that follow $(19,20)$. Numerous biochemical mediators of vascular permeability have been proposed, but, as of yet, no therapies specifically aimed at modulating cerebrovascular permeability have been used. Corticosteroids are used for acute therapy of cerebral edema, and, although the mode of action is not certain, it has been hypothesized that they may act by directly altering vascular permeability at the capillary bed level or by the inhibition of the production of permeability factors by tumor cells. Beneficial effects of corticosteroids have been demon- strated in patients with primary or metastatic brain tumors, but most clinical studies of craniocerebral trauma have failed to demonstrate efficacy.

Currently, nearly all forms of therapy for brain edema remain nonspecific and are directed at reducing intracranial pressure - the principal complication of brain edema - rather than reducing edema formation specifically. It is interesting to note that dexamethasone, used for the treatment of cerebral edema associated with neoplasia, has been shown to inhibit the biological activity of VEGF (21-23). Because dexamethasone is known to be efficacious in reducing the formation of brain edema in animal models of stroke, it is interesting to speculate that the observed protective effect of dexamethasone may be mediated through its action on VEGF.

With the exception of specialized cell types, such as epithelial cells in the choroid plexus, only diffuse expression of VEGF has been observed in adult brain (24). However, transient hypoxia/ischemia results in marked increase in VEGF and its receptors at both the mRNA and protein level. Under systemic hypoxia, for example, VEGF expression was seen in glial cells as well neurons in the hippocampus and dentate gyrus (24). Expression of VEGF and its receptors after occlusion of the MCA in the rat brain has been studied by a number of investigators (8-10). Enhanced VEGF expression was seen within the ischemic territory and was found to be localized in most cell types, including neurons and glia, as well as infiltrating macrophages. VEGF expression has been identified with cells associated with CNS inflammation, including microglial cells/macrophages (25) and reactive astrocytes, suggesting a central role in CNS inflammation (26). VEGF expression does not 
appear to be localized to endothelial cells in vivo after a hypoxic insult, although marked receptor expression is noted, suggesting a paracrine action under these conditions (24). In stroke studies, although the pattern and temporal profile of expression is dependent on the model as well as the techniques used for biochemical analysis, in general, expression of VEGF and its receptors occurs hours after onset of hypoxic-ischemia insult and persists for several days $(8,10)$. The time course for VEGF expression is consistent with the formation of cerebral edema and the inflammation associated with these models of stroke. Further, it has been shown that prolonged exposure to hypoxia results in a transient increase in VEGF that is maximal 1-2 days after onset of the hypoxic insult, and does not persist despite continued hypoxia (27).

VEGF is also known as vascular permeability factor, because of its ability to induce vascular leak (28). Some of the original assays used for the characterization of VEGF were based on vascular permeability enhancement to plasma protein, as demonstrated using the Miles assay. More recently, it has been shown that VEGF has a dramatic increase in hydraulic conductivity in isolated, perfused frog mesentery (2), confirming that VEGF has a specific action on microvessel permeability that is independent of any hemodynamic alterations that may be induced by VEGF. Although the relationship between the endothelial cell permeability enhancement and its mitogenic and angiogenic properties remain uncertain (28), VEGF and its endothelial cell receptors are overexpressed in a number of non-neoplastic pathologies. These are characterized by hyperpermeability to circulatory plasma proteins, including retinopathies, rheumatoid arthritis, psoriasis, and other inflammatory conditions. Numerous studies exist that provide corroborative evidence linking VEGF to the formation of edema. The high abundance of VEGF in brain tumors, together with the prevalence of peritumor edema has lead a number of groups to investigate the correlation between the extent of cerebral edema, often as determined from the MRI exam, with the levels of VEGF from tissues samples (29-32). All of these studies demonstrate an association between VEGF expression and vasogenic edema, suggesting a role for enhanced microvascular permeability effects of VEGF in the formation of cerebral edema. These studies cannot, however, distinguish cause from effect. Without the appropriate pharmacological antagonist, the contribution of VEGF to the formation of cerebral edema could not be verified.

The underlying mechanisms responsible for the reduction in cortical tissue damage seen in the present study remain unclear. Although it is possible that the observed effects could be mediated via a direct consequence of changes in vascular water flux across the blood-brain barrier, we have not measured vascular permeability directly. It should be stated that measurements of the extravasation of a particular solute (for example, albumin using Evans blue staining) are dependent upon a number of different variables and

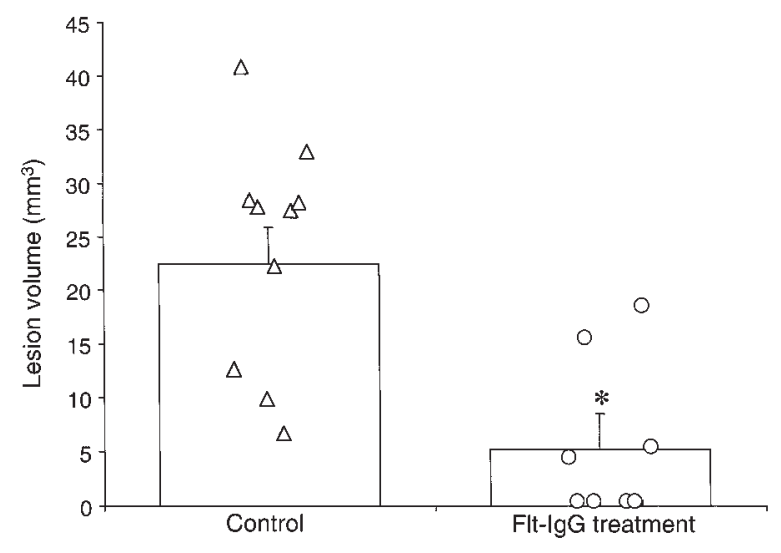

\section{Figure 6}

A single dose of mFlt(1-3)-IgG given 30 minutes before onset of ischemia affords a significant reduction in lesion volume. The lesion volume is defined as the region of single hyperintensity on the T2weighted MRI recorded 1 day after 30 minutes of ischemia with reperfusion. Values for individual animals are shown together with their means \pm SEM $\left({ }^{*} P<0.01\right)$.

are not a direct measure of permeability. Furthermore, although a number of investigators have demonstrated extravasation of Evans blue dye after focal ischemia, it has also been shown that albumin plays essentially no role in edema formation (33). Because the primary objective of the present study was to assess the pathophysiological consequences of VEGF antagonism, we chose to monitor brain edema directly using T2weighted MRI techniques. Other physiological actions of VEGF, for example vasodilation (34) and VEGFinduced hypotension (35), could confound interpretation of our experimental observations. Although they cannot be excluded as possible contributory mechanisms, hemodynamic perturbations during the occlusion period was not detected in our study.

In summary, we have shown that VEGF expression after transient ischemia in the brain contributes to the formation of cerebral edema. We have established that mFlt(1-3)-IgG treatment significantly reduces the acute appearance of cortical edema, and this is likely to offer long-term salvage of cortical tissue. Further work is required to identify the exact mechanisms responsible for the protection seen in this pathology, nevertheless, these results suggest a novel strategy for the management and prevention of cerebral edema associated with acute neurological disorders such as stroke and closed-head injury.

\footnotetext{
1. Betz, A.L. 1997. Vasogenic brain edema. In Primer on cerebrovascular diseases. K.M.A. Welch, L.R. Caplan, D.J. Reis, B.K. Siesjo, and B. Weir, editors. Academic Press. San Diego, CA. 156-159.

2. Bates, D.O., and Curry, F.E. 1996. Vascular endothelial growth factor increases hydraulic conductivity of isolated perfused microvessels. Am. J. Physiol. 271:2520-2528.

3. Bates, D.O., Lodwick, D., and Williams, B. 1999. Vascular endothelial growth factor and microvascular permeability. Microcirculation. 6:83-96. 4. Ferrara, N., et al. 1998. Vascular endothelial growth factor is essential for corpus luteum angiogenesis. Nat. Med. 4:336-340.

5. Senger, D.R., Connolly, D.T., Van de Water, L., Feder, J., and Dvorak, H.F. 1990. Purification and $\mathrm{NH}_{2}$-terminal amino acid sequence of guinea pig tumor-secreted vascular permeability factor. Cancer Res. 50:1774-1778.
} 
6. Monacci, W.T., Merrill, M.J., and Oldfield, E.H. 1993. Expression of vascular permeability factor/vascular endothelial growth factor in normal rat tissues. Am. J. Physiol. 264:995-1002.

7. Dor, Y., and Keschet, E. 1997. Ischemia-driven angiogenesis. Trends Cardiovasc. Med. 7:289-294.

8. Kovacs, Z., Ikezaki, K., Samoto, K., Inamura, T., and Fukui, M. 1996 VEGF and flt. Expression time kinetics in rat brain infarct. Stroke. 27:1865-1872.

9. Hayashi, T., Abe, K., Suzuki, H., and Itoyama, Y. 1997. Rapid induction of vascular endothelial growth factor gene expression after transient middle cerebral artery occlusion in rats. Stroke. 28:2039-2044.

10. Lennmyr, F., Ata, K.A., Funa, K., Olsson, Y., and Terent, A. 1998. Expression of vascular endothelial growth factor (VEGF) and its receptors (Flt1 and Flk-1) following permanent and transient occlusion of the middle cerebral artery in the rat. J. Neuropathol. Exp. Neurol. 57:874-882.

11. Issa, R., et al. 1999. Vascular endothelial growth factor and its receptor, $\mathrm{KDR}$, in human brain tissue after ischemic stroke. Lab. Invest. 79:417-425.

12. Nag, S., Takahashi, J.L., and Kilty, D.W. 1997. Role of vascular endothelial growth factor in blood-brain barrier breakdown and angiogenesis in brain trauma. J. Neuropathol. Exp. Neurol. 56:912-921.

13. Hayashi, T., Abe, K., and Itoyama, Y. 1998. Reduction of ischemic damage by application of vascular endothelial growth factor in rat brain after transient ischemia. J. Cereb. Blood Flow Metab. 18:887-895.

14. de Vries, C., et al. 1992. The fms-like tyrosine kinase, a receptor for vascular endothelial growth factor. Science. 255:989-991.

15. Aiello, L.P., et al. 1995. Suppression of retinal neovascularization in vivo by inhibition of vascular endothelial growth factor (VEGF) using soluble VEGF-receptor chimeric proteins. Proc. Natl. Acad. Sci. USA. 92:10457-10461.

16. Chen, S.T., Hsu, C.Y., Hogan, E.L., Maricq, H., and Balentine, J.D. 1986. A model of focal ischemic stroke in the rat: reproducible extensive cortical infarction. Stroke. 17:738-743.

17. Franklin, K.B.J., and Paxinos, G.T. 1997. The mouse brain in stereotaxic coordinates. Academic Press. San Diego, CA. 304pp.

18. van Lookeren Campagne, M., et al. 1999. Secondary reduction in the apparent diffusion coefficient of water, increase in cerebral blood volume and delayed neuronal death following middle cerebral artery occlusion and early reperfusion in the rat. J. Cereb. Blood Flow Metab. In press.

19. Klatzo, I. 1983. Disturbances of the blood-brain barrier in cerebrovascular disorders. Acta Neuropathol. (Berl.). 8:81-88.

20. Hariri, R.J. 1994. Cerebral edema. Neurosurg. Clin. N. Am. 5:687-706.

21. Nauck, M., Karakiulakis, G., Perruchoud, A.P., Papakonstantinou, E., and Roth, M. 1998. Corticosteroids inhibit the expression of the vascular endothelial growth factor gene in human vascular smooth muscle cells. Eur. J. Pharmacol. 341:309-315.
22. Heiss, J.D., et al. 1996. Mechanism of dexamethasone suppression of brain tumor-associated vascular permeability in rats. Involvement of the glucocorticoid receptor and vascular permeability factor. J. Clin. Invest. 98:1400-1408.

23. Bruce, J.N., et al. 1987. Vascular permeability induced by protein product of malignant brain tumors: inhibition by dexamethasone. J. Neurosurg. 67:880-884.

24. Marti, H.H., and Risau, W. 1998. Systemic hypoxia changes the organspecific distribution of vascular endothelial growth factor and its receptors. Proc. Natl. Acad. Sci. USA. 95:15809-15814.

25. Plate, K.H., Beck, H., Danner, S., Allegrini, P.R., and Wiessner, C. 1999. Cell type specific upregulation of vascular endothelial growth factor in an MCAocclusion model of cerebral infarct. J. Neuropathol. Exp. Neurol. 58:654-666.

26. Proescholdt, M.A., et al. 1999. Vascular endothelial growth factor (VEGF) modulates vascular permeability and inflammation in rat brain. J. Neuropathol. Exp. Neurol. 58:613-627.

27. Patt, S., et al. 1998. Upregulation of vascular endothelial growth factor in severe chronic brain hypoxia of the rat. Neurosci. Lett. 252:199-202.

28. Dvorak, H.F., Nagy, J.A., Feng, D., Brown, L.F., and Dvorak, A.M. 1999. Vascular permeability factor/vascular endothelial growth factor and the significance of microvascular hyperpermeability in angiogenesis. Curr. Top. Microbiol. Immunol. 237:97-132.

29. Strugar, J.G., Criscuolo, G.R., Rothbart, D., and Harrington, W.N. 1995. Vascular endothelial growth/permeability factor expression in human glioma specimens: correlation with vasogenic brain edema and tumorassociated cysts. J. Neurosurg. 83:682-689.

30. Criscuolo, G.R. 1993. The genesis of peritumoral vasogenic brain edema and tumor cysts: a hypothetical role for tumor-derived vascular permeability factor. Yale J. Biol. Med. 66:277-314.

31. Goldman, C.K., et al. 1997. Brain edema in meningiomas is associated with increased vascular endothelial growth factor expression. Neurosurgery. 40:1269-1277.

32. Kalkanis, S.N., Carroll, R.S., Zhang, J., Zamani, A.A., and Black, P.M. 1996. Correlation of vascular endothelial growth factor messenger RNA expression with peritumoral vasogenic cerebral edema in meningiomas. J. Neurosurg. 85:1095-1101.

33. Menzies, S.A., Betz, A.L., and Hoff, J.T. 1993. Contributions of ions and albumin to the formation and resolution of ischemic brain edema. $J$. Neurosurg. 78:257-266.

34. Ku, D.D., Zaleski, J.K., Liu, S., and Brock, T.A. 1993. Vascular endothelial growth factor induces EDRF-dependent relaxation in coronary arteries. Am. J. Physiol. 265:586-592.

35. Yang, R, et al. 1996. Effects of vascular endothelial growth factor on hemodynamics and cardiac performance. J. Cardiovasc. Pharmacol. 27:838-844. 